\title{
Overexpression of a dominant-negative allele of YPT1 inhibits growth and aspartyl protease secretion in Candida albicans
}

\author{
Samuel A. Lee, ${ }^{1,2}$ Yuxin Mao, ${ }^{1}$ Zimei Zhang $^{1}$ and Brian Wong ${ }^{1,2}$ \\ Author for correspondence: Brian Wong. Tel: +1 203937 3446. Fax: +1 2039373476 \\ e-mail: brian.wong@yale.edu
}

1 Infectious Diseases Section, Yale University School of Medicine, New Haven, CT 06520, USA

2 Infectious Diseases Section, VA Connecticut Healthcare System, 950 Campbell Ave, Bldg $8(111-1)$, West Haven, CT 06516, USA
To investigate the pre-Golgi secretion pathway in the pathogenic yeast Candida albicans, we cloned the C. albicans homologue of the Saccharomyces cerevisiae protein secretion gene YPT1. The $C$. albicans YPT1 ORF contained a 624 bp intronless ORF encoding a deduced protein of 207 aa and 2.3 kDa. This deduced protein was $77 \%$ identical to $S$. cerevisiae Ypt1 protein (Ypt1p) and it contained GTP-binding domains that are conserved among all known ras-like GTPases. Multicopy plasmids containing C. albicans YPT1 complemented the temperature-sensitive S. cerevisiae ypt1 (A136D) mutation. One chromosomal YPT1 allele in C. albicans CAI4 was readily disrupted by homologous gene targeting, but attempts to disrupt the second allele yielded no viable null mutants. Since this suggested that $C$. albicans YPT1 may be essential, a mutant ypt1 allele was constructed encoding the amino acid substitution analogous to the $\mathbf{N} 121$ I substitution in a known trans-dominant inhibitor of $S$. cerevisiae Ypt1p. Next, a GAL1-regulated plasmid was used to express the mutant ypt1(N121I) allele in C. albicans CAl4. Ten of 11 transformants tested grew normally in glucose and poorly in galactose, and plasmid curing restored growth to wild-type levels. When these transformants were incubated in galactose, secretion of aspartyl proteinase (Sap) was inhibited and membranebound secretory vesicles accumulated intracellularly. These results imply that C. albicans YPT1 is required for growth and protein secretion, and they confirm the feasibility of using inducible dominant-negative alleles to define the functions of essential genes in C. albicans.

Keywords: Candida albicans, protein secretion, vesicle trafficking, YPT1

\section{INTRODUCTION}

Candida albicans has emerged as a significant opportunistic pathogen since the routine use of broadspectrum antibiotics and immunosuppressive drugs has become widespread. Candida species have become the fourth most commonly isolated micro-organism from the bloodstream of hospitalized patients in the United States (Emori \& Gaynes, 1993; Jarvis, 1995). Although C. albicans is an increasingly important pathogen, an incomplete understanding of Candida pathogenesis and cellular biology has restricted the ability to diagnose and treat invasive candidiasis. A number of C. albicans

The GenBank accession number for the $C$. albicans YPT1 sequence reported in this paper is AF330211. virulence factors, such as the secreted aspartyl proteases (Sap) (Hoegl et al., 1996; Hube et al., 1997; Sanglard et al., 1997), extracellular phospholipases (Ghannoum, 2000), Int1p (Gale et al., 1998) and the outer mannoprotein Hwp1 (Staab et al., 1999), have been identified, and many of these are found either on the cell surface or in the extracellular environment. However, little is known about the means by which these virulenceassociated proteins are processed and secreted.

Therefore, our long-term goal is to characterize key steps in the processing and secretion of virulenceassociated proteins in C. albicans. In an earlier study, Mao et al. (1999) showed that the C. albicans homologue of the essential Saccharomyces cerevisiae secretion pathway gene SEC4 encodes a ras-like GTPase that is required for growth, secretion of Sap and fusion of postGolgi secretory vesicles to the plasma membrane. In $S$. 
cerevisiae, the essential gene YPT1 encodes another raslike GTPase that regulates a key step in pre-Golgi vesicle transport (Novick \& Brennwald, 1993). S. cerevisiae ypt 1 mutants are defective in protein secretion and display intracellular accumulation of vesicles and elongated structures resembling endoplasmic reticulum (ER) (Segev et al., 1988). In the present study, we cloned the C. albicans homologue of S. cerevisiae YPT1 and we used molecular genetic approaches to study the function of this gene and to examine its effects on Sap secretion.

\section{METHODS}

Strains and media. C. albicans strains SC5314 (wild-type) and

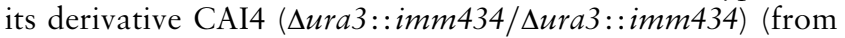
W. Fonzi, Georgetown University) were grown at $30{ }^{\circ} \mathrm{C}$ in YPD ( $1 \%$ yeast extract, $2 \%$ peptone, $2 \%$ glucose), minimal glucose $[0.67 \%$ yeast nitrogen base without amino acids (YNB), $2 \%$ glucose] or minimal galactose $(0.67 \%$ YNB, $2 \%$ galactose). S. cerevisiae strain NSY 161 (MAT $\alpha$, his4 ura3-52 $y p t 1-A 136 D$ ) was from N. Segev (University of Chicago). Sap secretion by C. albicans was examined in $0.34 \%$ YNB without ammonium sulfate and with $0 \cdot 2 \%$ bovine serum albumin (BSA), $0 \cdot 2 \%$ yeast extract and either $2 \%$ glucose (glucoseBSA-YE) or $2 \%$ galactose (galactose-BSA-YE). Uracil auxotrophs were selected on 5-fluoroorotic acid (FOA) medium (minimal glucose, $0 \cdot 1 \mathrm{mg}$ uridine $\mathrm{ml}^{-1}$ and $0.7 \mathrm{mg} \mathrm{FOA} \mathrm{ml}^{-1}$ ). Plasmids were expanded in Escherichia coli $\mathrm{DH} 5 \alpha$ cells grown in LB medium plus ampicillin $\left(100 \mu \mathrm{g} \mathrm{ml}^{-1}\right)$ at $37^{\circ} \mathrm{C}$. Solid media were prepared by adding $2 \%$ agar.

Libraries and plasmids. Plasmid p5921, which contains a his GURA3-hisG selectable marker, and a library of C. albicans SC5314 genomic DNA fragments in $\lambda$ GEM-12 were obtained from W. Fonzi (Georgetown University). Plasmid YM1, which contains the C. albicans GAL1 promoter, has been previously described (Mao et al., 1999). Plasmid YEp352 was obtained from J. Perfect (Duke University). pBluescript II SK was from Stratagene. Plasmid SFNB490 (which contains S. cerevisiae YPT1) was obtained from P. Novick (Yale University).

Isolation and analysis of C. albicans YPT1. A 305 bp partial C. albicans gene fragment homologous to $S$. cerevisiae YPT1 was identified by searching the Stanford C. albicans genome sequencing database, and a $298 \mathrm{bp}$ portion of this sequence was amplified from C. albicans SC5314 genomic DNA and labelled with ${ }^{32} \mathrm{P}$ by PCR, using Taq DNA polymerase and primers P24 (5'-CGAGTGTATATGTGTCGTCAGC-3') and M11 (5'-TCTCTTTGTATGCTTGTACCCG-3'). This PCR product was used to screen a $\lambda$ library of C. albicans genomic DNA and positive plaques were identified by autoradiography and purified. The gene of interest was localized to a $2 \cdot 8 \mathrm{~kb}$ $\mathrm{XbaI}$ restriction fragment which was ligated into pBluescript SK II to generate pBSYP. Standard methods were used for restriction mapping, subcloning, Southern hybridization, DNA sequencing and transformation of $S$. cerevisiae mutants.

Genomic Southern blots were probed with a $210 \mathrm{bp}{ }^{32} \mathrm{P}-$ labelled portion of C. albicans YPT1 that encodes a part of $C$. albicans Ypt1p that is analogous to the C-terminal hypervariable region of $S$. cerevisiae Ypt1p (Brennwald \& Novick, 1993). The probe was amplified from C. albicans SC5314 genomic DNA by PCR, using primers Hyp1 (5'-CTATGGCAAGACAAATCAAAGCCC-3') and HCC (5'-CAACTAATCTTCCCCTGATATTTC-3'). This portion of YPT1 was used to minimize cross-hybridization to genes encoding other ras-related GTP-binding proteins. Chromosomal mapping was performed by B. B. Magee (University of Minnesota), as described by Chu et al. (1993).

Targeted disruption of C. albicans YPT1. To disrupt both chromosomal alleles of $C$. albicans YPT1, it was first necessary to construct a selectable $y p t 1 \Delta:$ : hisG-URA3-his $G$ gene disruption cassette. The first step was to use Taq DNA polymerase and primers A1 (5'-GAAGATCTAGATAAACAGTCGTGTATAAGATG-3') and A2 (5'-CGCGGATCC GTTGCTGTCTGACTTATCGG-3') to amplify $4 \overline{72 \text { bp of } 5^{\prime}}$ untranslated C. albicans YPT1 DNA from pBSYP and to add $B g l$ II and BamHI restriction sites (underlined). The PCR product was digested with $B a m \mathrm{HI}$ and $B g l \mathrm{II}$ and ligated into the BglII site in p5921, which yielded pL1. Next, primers Z001 (5'-GAAGATCTTGCTGATGCCTTGGACATTCC-3') and Z2 (5'-CGCGGATCCAATAAGAACATTACCTATTTAAAACAAC-3') were used to amplify 351 bp of DNA from the $3^{\prime}$ end of the C. albicans YPT1 ORF in pBSYP and to add BglII and Bam HI restriction sites (underlined). This PCR product was digested with $B a m H I$ and $B g l$ II and ligated into the BamHI site in pL1, which yielded pL2. Proper orientation of the inserts was confirmed at each step by PCR and restriction analysis and accuracy of the final construct was confirmed by DNA sequencing.

To disrupt the first chromosomal YPT1 allele, pL2 was digested with BamHI and BglII, and $5 \mu \mathrm{g}$ of the linearized $y p t 1 \Delta:$ : his G-URA3-his G gene disruption cassette was introduced into $C$. albicans CAI4 by the lithium acetate method (Ausubel et al., 1987). Uracil prototrophs were selected on minimal glucose and purified, and genomic DNA was extracted by vortexing with glass beads. Two methods were used to determine if homologous integration of the $y p t 1 \Delta:$ : hisG-URA3-hisG gene disruption cassette at the YPT1 locus had occurred. First, allele-specific PCR with primers derived from the C. albicans YPT1 locus, P1 (5'-CGATCCAGCCAATTCATTAC-3'), and from the his $G$ region of p5921, G2 (5'-GCGCGGCGCGACTTCGACAGAACC-3'), was used to determine if the linearized $y p t 1 \Delta:$ : his G-URA3his $G$ gene disruption cassette had integrated homologously within a YPT1 locus. Also, PCR with primers A22 $\left(5^{\prime}\right.$ CTTCTACAGTCGAACAGAGAAG-3') and HCC (5'-CAACTAATCTTCCCCTGATATTTC-3') was used to detect changes in the sizes of the chromosomal YPT1 loci. Second, C. albicans genomic DNA was digested with XmnI and transferred to nylon membranes. The membranes were hybridized in $4 \times$ SSC $(1 \times$ SSC is $0 \cdot 15 \mathrm{M} \mathrm{NaCl}, 0.015 \mathrm{M}$ sodium citrate $)$ at $65{ }^{\circ} \mathrm{C}$ with a $235 \mathrm{bp}{ }^{32} \mathrm{P}$-labelled PCR product generated from pBSYP with primers NP01 (5'-ACGGGATATTACTCATGGTGACC-3') and OUT1 (5'-CCAACAGAATGACGGAGAATAC- $\left.3^{\prime}\right)$, after which the membranes were washed in $0.2 \times \mathrm{SSC} / 0.1 \% \mathrm{SDS}$ at $65^{\circ} \mathrm{C}$ and analysed using the DIG DNA detection kit (Boehringer Mannheim).

To disrupt the second YPT1 allele, selected C. albicans YPT1/ypt1s: :hisG-URA3-hisG mutants were expanded in YPD to permit loss of $U R A 3$ by cis recombination between the flanking his $G$ repeats, uracil auxotrophs were selected on FOA medium and the genotypes of these strains were determined by PCR and Southern hybridization as described

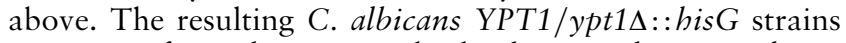
were transformed again with the linearized $y p t 1 \Delta:$ : his GURA3-hisG gene disruption cassette, uracil prototrophs were selected and their genotypes were analysed by PCR and by Southern hybridization.

Construction of plasmids pYPT1, pN121I and pD124N. Mutant ypt1 alleles encoding an Asn to Ile substitution at position 121 (N121I) and an Asp to Asn substitution at position 124 


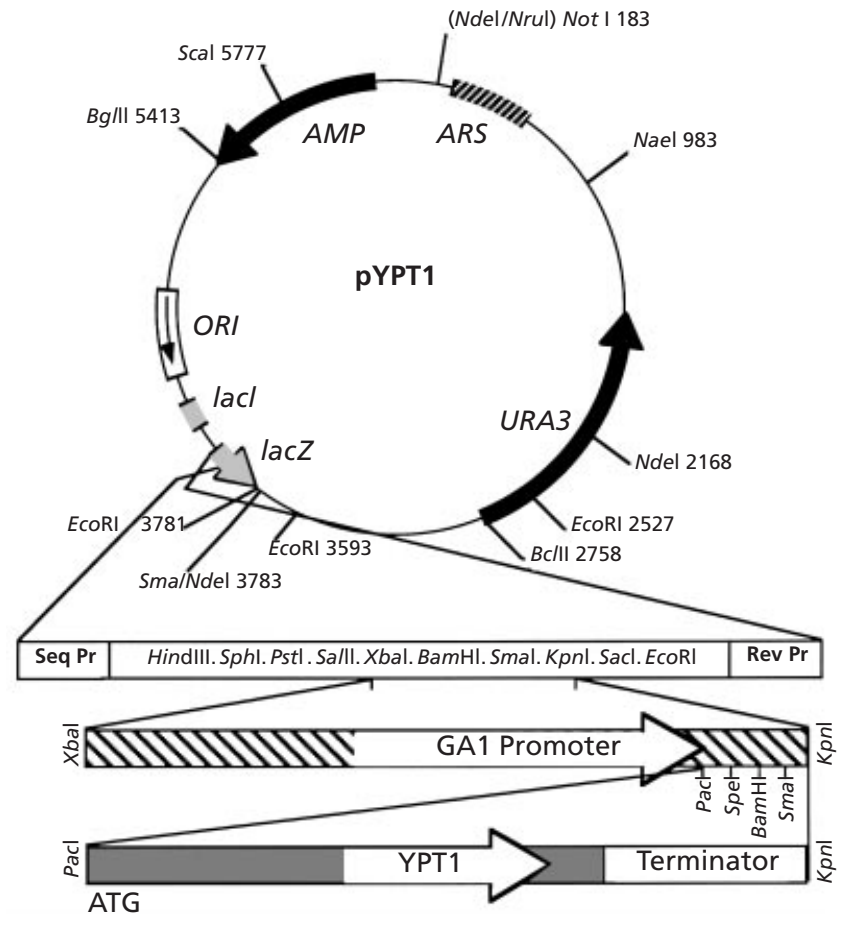

Fig. 1. Plasmid pYPT1 was constructed by inserting wild-type C. albicans YPT1 into the multiple-cloning site of pYM1, immediately downstream of the regulable GAL1 promoter. The C. albicans ypt1(N121I) allele was generated by PCR-based sitedirected mutagenesis and ligated into the multiple-cloning site of pYM1, to yield pN121I. pD124N is identical to pYPT1 and pN121I, except the ypt1(D124N) allele was used.

(D124N) of C. albicans Ypt1p were constructed using the QuickChange PCR-based site-directed mutagenesis method (Stratagene). The N121I mutation was generated with Pfu DNA polymerase, pBSYP as the template, and oligonucleotides N121I5 (5'-CCGGTGTTGGGAAAAATTGTTTATTATTGCGTTTTG-3') and N121I3 (5'-CAAAACGCAATAATAAACAATTTTTCCCAACACCGG-3') as primers. The D124N mutation was generated in analogous fashion using oligonucleotides D124N5 (5'-GTTGGTAATAAGGCTAATTTGTCTGATAAAAAAATC-3') and D124N3 (5'GATTTTTTTATCAGACAAATTAGCCTTATTACCAAC-3'). Primers 5P1 (5'-CCTTAATTAAATGAATAACGAATACGAC-3') and 3K1 (5'-GGGGTACCAAATAAGAACATTACCTATT-3') were used to amplify the wild-type YPT1, $y p t 1(\mathrm{~N} 121 \mathrm{I})$ and $y p t 1(\mathrm{D} 124 \mathrm{~N})$ ORFs, and the $1.0 \mathrm{~kb}$ PCR products were cloned into the PacI and SpeI sites of the GAL1regulated $C$. albicans expression plasmid pYM1, yielding plasmids pYPT1, pN121I and pD124N, respectively (Fig. 1). The accuracy of these DNA constructs was confirmed by DNA sequencing.

Effects of pYPT1, pN121I and pD124N in C. albicans. Plasmids pYPT1, pN121I and pD124N were introduced into C. albicans CAI4 by the lithium acetate method and uracil prototrophs were selected on minimal glucose. Growth was assessed both on glucose and galactose agar, and by measuring $\mathrm{OD}_{600}$ at intervals after strains SL21 (CAI4 transformed with pN121I), SL22 (CAI4 transformed with pYPT1) and control strain SC5314 were washed and transferred to fresh minimal glucose or minimal galactose media.
Whether differences in growth rates were plasmid-mediated was examined by plasmid curing. Representative $\mathrm{ura}^{+}$transformants were expanded for 40 generations in liquid YPD medium and $u \mathrm{ra}^{-}$clones were selected and purified on FOA plates. Growth of these $u r a^{-}$clones was then compared to growth of the $u r a^{+}$transformants on minimal glucose and minimal galactose.

Sap expression was induced by growing the C. albicans transformants to stationary phase in minimal glucose, after which the cells were washed and resuspended at $\mathrm{OD}_{600} 10$ in glucose-BSA-YE or galactose-BSA-YE. The cell suspensions were shaken at $30^{\circ} \mathrm{C}$ and cell-free supernatants obtained after 2-24 h were tested for residual BSA by SDS-PAGE with Coomassie blue staining and for immunoreactive Sap by Western blotting, using polyclonal rabbit antibodies to Sap (from N. Agabian, University of California at San Francisco).

The ultrastructural morphology of C. albicans cells overexpressing $y p t 1(\mathrm{~N} 121 \mathrm{I})$ was determined by transferring glucose-grown SL21, SL22 and SC5314 cells to minimal glucose or minimal galactose media for $6 \mathrm{~h}$. Cells from $10 \mathrm{ml}$ of culture were harvested by centrifugation, fixed in $3 \%$ glutaraldehyde $/ 0 \cdot 1 \mathrm{M}$ sodium cacodylate $(\mathrm{pH} 6 \cdot 8)$ overnight at $4{ }^{\circ} \mathrm{C}$, washed with $0 \cdot 1 \mathrm{M}$ sodium cacodylate and post-fixed in $1 \% \mathrm{OsO}_{4}$ for $1-2 \mathrm{~h}$. The fixed cells were embedded in Epox $812 \mathrm{x}$ and polymerized at $60^{\circ} \mathrm{C}$ for $48 \mathrm{~h}$, sectioned, stained with lead citrate/uranyl acetate and examined with a Philips 300 electron microscope.

\section{RESULTS}

\section{Properties of C. albicans YPT1}

DNA sequencing of the $2 \cdot 8 \mathrm{~kb} \mathrm{XbaI}$ insert in pBSYP revealed the presence of a $624 \mathrm{bp}$ intronless ORF whose deduced protein product was $77 \%$ identical with $S$. cerevisiaeYpt1p and included GTP-binding regions that are highly conserved in all members of the Sec/Ypt/Rab family of ras-like GTPases (Brennwald \& Novick, 1993; Novick \& Brennwald, 1993). In addition, this deduced C. albicans protein (Ypt1p) was identical to $S$. cerevisiae Ypt1p at five positions where single amino acid substitutions are known to cause dominant-negative or temperature-sensitive mutations (Fig. 2) (Schmitt et al., 1986; Jones et al., 1995; Segev \& Botstein, 1987; Becker et al., 1991; Jedd et al., 1995).

To determine if the C. albicans YPT1 homologue was also functionally homologous to $S$. cerevisiae YPT1, the $2.8 \mathrm{~kb} \mathrm{XbaI}$ fragment from pBSYP was ligated into YEp352 and the resulting plasmid (pYEYP) was transformed into S. cerevisiae strain NSY161, which contains the temperature-sensitive $y p t 1-A 136 D$ allele (Jedd et al., 1995). Saccharomyces transformed with pYEYP or with S. cerevisiae YPT1 in Yep 352 grew well at $37^{\circ} \mathrm{C}$, but the transformants bearing the YEp352 alone did not (Fig. 3).

A portion of C. albicans YPT1 encoding a region analogous to the C-terminal hypervariable domain of $S$. cerevisiae Ypt1p hybridized to single bands in SacI, NotI, BamHI, EcoRV, XhoI and XbaI digests of C. albicans CAI4 genomic DNA (data not shown). Chromosomal mapping localized C. albicans YPT1 on Sfil fragment S of chromosome 1 (B. Magee, personal communication). 


\begin{tabular}{|c|c|c|c|}
\hline \multirow[b]{2}{*}{$\mathrm{CA}$} & & & \multirow{3}{*}{$\begin{array}{l}\text { Fig. 2. The deduced Ypt1ps C. albicans (CA) } \\
\text { and S. cerevisiae (SC) were } 77 \% \text { identical (:). } \\
\text { Conserved GTP binding }\left({ }^{*}\right), \text { GTPase effector }\end{array}$} \\
\hline & 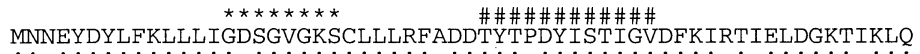 & 60 & \\
\hline $\mathrm{SC}$ & MUSEYDYL & 60 & \\
\hline $\mathrm{CA}$ & $\begin{array}{l}* * * * * * * \\
\text { IWDTAGQERFRTITSSYYRGAHGIIIVYDVTDQESFNNVKQWLQEIDRYATGGVMKLLVG }\end{array}$ & 120 & $\begin{array}{l}(\#) \text { and membrane attachment }(+) \text { domains } \\
\text { are shown as are positions in S. cerevisiae }\end{array}$ \\
\hline $\mathrm{SC}$ & 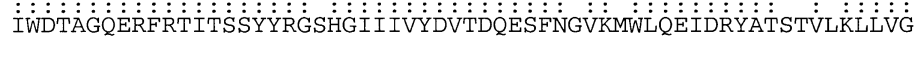 & 120 & $\begin{array}{l}\text { are snown, as are positions in s. cerevisiae } \\
\text { Ypt1p where known substitutions cause }\end{array}$ \\
\hline $\mathrm{CA}$ & $\begin{array}{l}* * * * \\
\text { NKADLSDKKIVEYTAAKEFADALDIPFLETSALSSTNVEQAFYTMARQIKAQMTNNANAG }\end{array}$ & 180 & $\begin{array}{l}\text { temperature-sensitive mutations }(\mathrm{T} 40 \rightarrow \mathrm{K} \text {, } \\
\mathrm{D} 44 \rightarrow \mathrm{N} \text { and } \mathrm{A} 136 \rightarrow \mathrm{D} ; \uparrow), \text { dominant- }\end{array}$ \\
\hline $\mathrm{SC}$ & 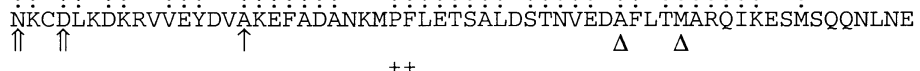 & 180 & $\begin{array}{l}\text { negative mutations }(\mathrm{N} 121 \rightarrow \mathrm{I} \text { and } \mathrm{D} 124 \rightarrow \mathrm{N} \text {; } \\
\Uparrow) \text { and temperature-sensitive suppression of }\end{array}$ \\
\hline $\mathrm{CA}$ & NAANAKGKSNVNLRGESLTSNQSNSCC & 207 & dominant $\mathrm{N} 121 \rightarrow \mathrm{I}$ mutation $(\mathrm{A} 161 \rightarrow \mathrm{V}$ \\
\hline SC & 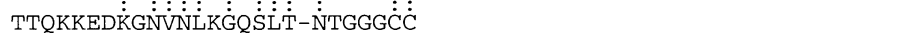 & 206 & \\
\hline
\end{tabular}

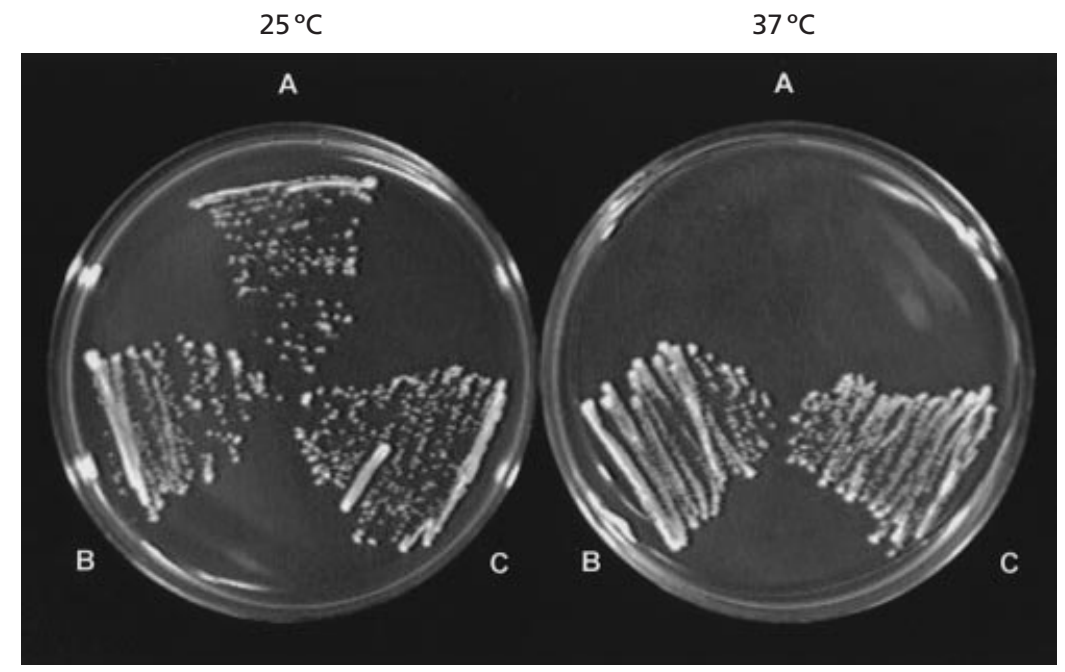

Fig. 3. Complementation of the $S$. cerevisiae ypt1-A136D temperature-sensitive mutant. Multicopy plasmids (YEp352) containing $S$. cerevisiae YPT1 (B) and C. albicans YPT1 (C) conferred on a temperature-sensitive Sc ypt1 mutant the ability to grow at $37^{\circ} \mathrm{C}$, but YEp352 alone (A) did not.

\section{Targeted disruption of C. albicans YPT1}

Since the results described above indicated that $C$. albicans YPT1 is present in a single copy per haploid genome, we next attempted to construct C. albicans ypt1 null mutants by homologous gene targeting. When the linearized insert from pL2 (which contains the $y p t 1 \Delta:$ : hisG-URA3-hisG gene disruption cassette) was introduced into C. albicans strain CAI4, homologous gene replacement was demonstrated by Southern analysis and allele-specific PCR in 19 of 20 transformants analysed (Fig. 4).

Initial efforts to identify YPT1/ypt1 $::$ his $G$ strains among FOA-resistant clones derived from two $C$. albicans YPT1/ypt1A: hisG-URA3-hisG mutants were unsuccessful. Twenty of 20 colonies analysed were indistinguishable from C. albicans CAI4 by genomic Southern hybridization and/or allele-specific PCR. Since this suggested that interchromosomal recombination between the mutant $y p t 1 \Delta:$ : hisG-URA3-his G allele and the remaining wild-type YPT1 allele was much more frequent than intrachromosomal recombination between the his $G$ repeats flanking the URA3 marker, we used PCR to screen 10 pools containing a total of 120 FOA-resistant colonies for his $G$ DNA. his $G$ DNA was identified in six pools and PCR screening of the individual members of two positive pools identified two individual clones containing hisG DNA (strains T7F4 and T8F11). Southern hybridization and allele-specific PCR confirmed the YPT1/ypt1A: :hisG genotype in both of these strains (Fig. 4).

Strain T7F4 was transformed again with the linearized $y p t 1 \Delta:$ : hisG-URA3-hisG gene disruption cassette. Twenty of 20 transformants derived from strain T7F4 were found by Southern hybridization and allele-specific PCR to retain one wild-type YPT1 allele. Twelve of the transformants demonstrated simple allelic replacement of the $y p t 1 \Delta:: h i s G$ allele with gene disruption cassette DNA and eight revealed more complex integration events. When no null mutants were found among the T7F4 transformants, we also transformed strain T8F11 with the same $y p t 1 \Delta:$ : hisG-URA3-hisG gene disruption cassette. Ten of 10 transformants analysed were found by allele-specific PCR and Southern hybridization to retain one wild-type YPT1 allele (Fig. 4).

\section{Effects of ypt1(N121I) overexpression on growth and viability}

Since the results above suggested, but did not prove that C. albicans YPT1 might be an essential gene, we used an alternative approach to study the functions of $C$. albicans YPT1. One strategy that is often used to 
(a)

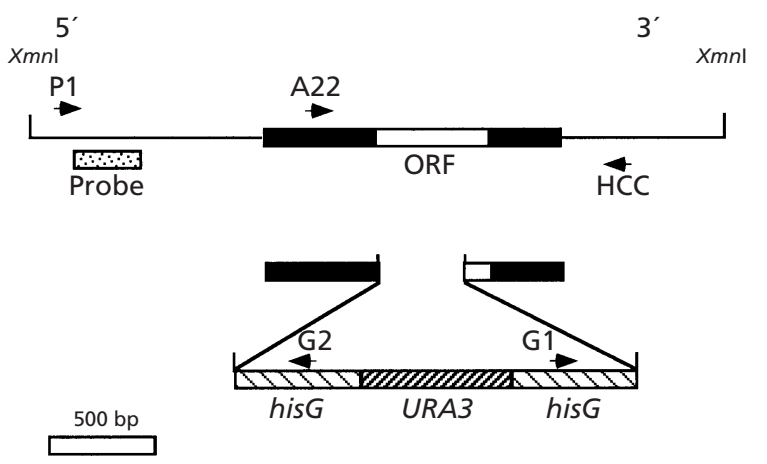

(c)

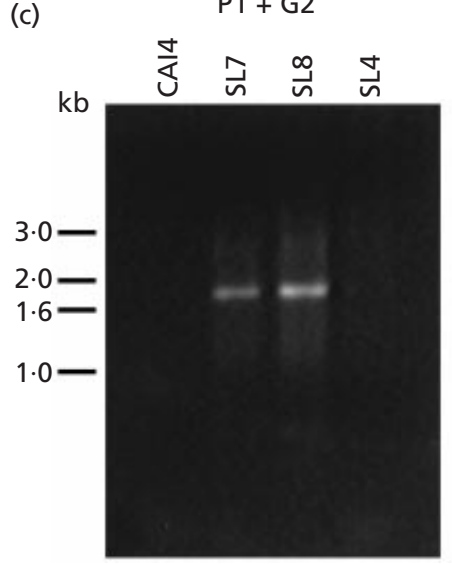

(b)

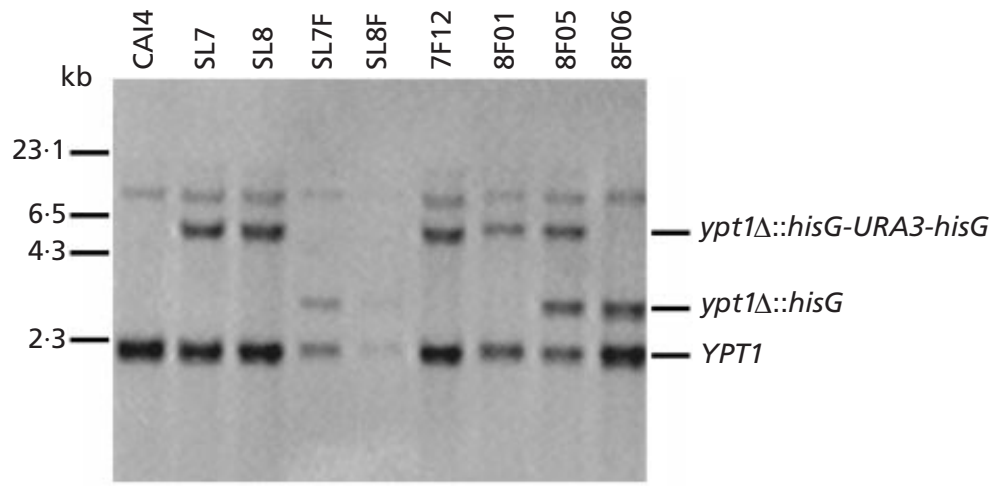

$\mathrm{A} 22+\mathrm{HCC}$

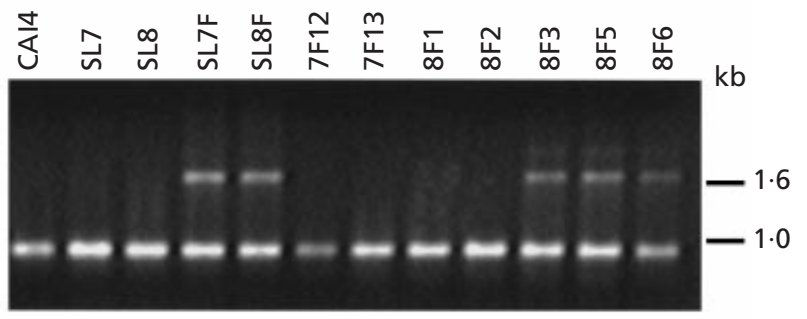

Fig. 4. Targeted disruption of $C$. albicans YPT1. (a) The $C$. albicans YPT1 gene locus is shown (top). A gene disruption cassette (bottom) was constructed by ligating PCR products with $5^{\prime}$ Bg/ll and 3' BamH1 restriction sites generated from each flanking region of C. albicans YPT1 to a hisG-URA3-hisG cassette in p5921. (b) Southern hybridization was performed on $\mathrm{Xmnl}$ digests of genomic DNA from 20 transformants using a radiolabelled probe to a region upstream of C. albicans YPT1. Since Xmnl yields YPT1 on a $2 \cdot 2 \mathrm{~kb}$ fragment and does not cut within the ypt1D::hisG-URA3-hisG gene disruption cassette, the probe hybridizes to a $2.2 \mathrm{~kb} X m n l$ fragment from wild-type YPT1 and a $5.8 \mathrm{~kb} X m n l$ fragment if the cassette integrates homologously into the YPT1 gene locus. Homologous gene replacement was confirmed by Southern analysis and allele-specific PCR in 19 of 20 transformants analysed (SL7, SL8). After 5-FOA selection, the resulting $C$. albicans ura3 $\triangle Y P T 1 / y p t 1 \Delta::$ hisG strains (SL7F, SL8F) were again transformed with linearized gene disruption cassette DNA. Twenty transformants derived from strain T7F4 were analysed by Southern hybridization after digestion with Xmnl. All 20 transformants retained one wild-type YPT1 allele. Twelve of these demonstrated simple allelic replacement of the ypt1A::hisG allele with gene disruption cassette DNA (7F12) and eight revealed more complex integration events (represented by 8F05). Analysis of another 10 transformants from strain T8F11 again showed that they all retained one wild-type YPT1 allele (8F01, 8F05, 8F06). (c) When C. albicans CAI4 was transformed with the linearized gene disruption cassette, PCR with primers $\mathrm{P} 1$ and G2 generated a product only if the gene disruption cassette integrated homologously (SL7 and SL8, but not wild-type CAI4 or ectopic integrant SL4). Also, primers A22 and HCC generated a $1.2 \mathrm{~kb}$ PCR product from wild-type YPT1, no additional product from the large ypt1 $\Delta::$ hisG-URA3-hisG cassette (SL7 and SL8) and a $1.6 \mathrm{~kb}$ product when recombination between flanking hisG repeats generated a smaller ypt1 $\Delta:$ :hisG allele (SL7F, SL8F). When SL7F or SL8F was transformed again with ypt1 $8 \mathrm{F02}$ ) and ectopic (8F03, 8F05, 8F06) integrations were observed. However, all transformants analysed retained at least one wild-type YPT1 allele.

examine the functions of essential genes in $S$. cerevisiae and other organisms is to determine the phenotypic consequences of overexpression of dominant-negative alleles. Because C. albicans Ypt1p shares some key structural features with $S$. cerevisiae Ypt1p and other Rab-family GTPases, we used this approach to analyse the functions of C. albicans YPT1. When Asn-121 of S. cerevisiae Ypt1p is replaced by Ile or when Asp-124 is replaced with Asn, the resulting proteins function as trans-dominant inhibitors of YPT1 function (Schmitt et al., 1986; Jones et al., 1995). Since C. albicans YPT1 encodes Asn and Asp in the analogous positions (Fig. 2), we constructed mutant $C$. albicans ypt1 alleles encoding the N121I and the D124N substitutions and we used a GAL1-regulated expression plasmid to express these alleles in $C$. albicans. 


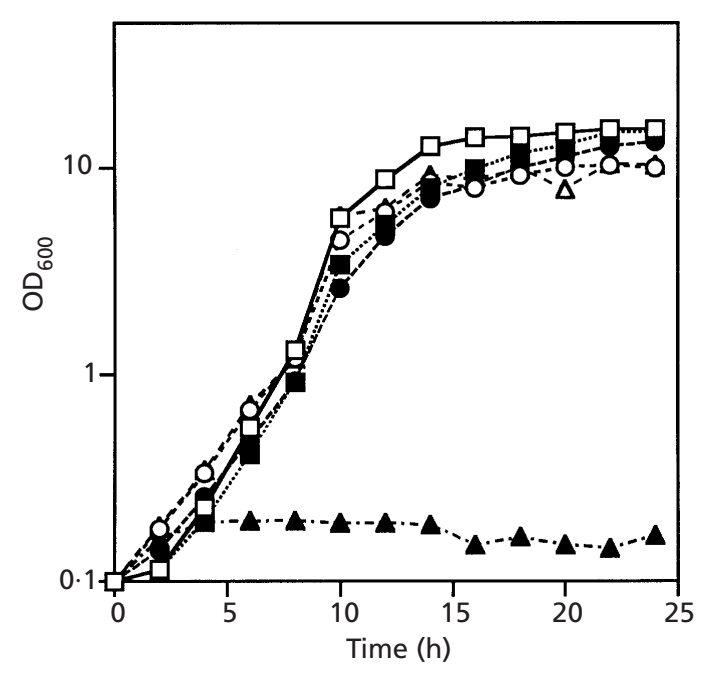

Fig. 5. Effects of ypt1(N121I) overexpression on growth. Wildtype C. albicans (SC5314) and a C. albicans pYPT1-transformant grew well in minimal glucose (Glu) and minimal galactose (Gal). A representative $C$. albicans pN121I-transformant also grew well in minimal glucose, but it grew very slowly in minimal galactose. $\square$, SC5314/Glu;, SC5314/Gal; O, pYPT1/Glu; pYPT1/Gal; $\triangle$, pN121//Glu; $\boldsymbol{\Delta}$, pN121//Gal.

Multiple attempts to introduce pD124N into C. albicans yielded only four $\mathrm{ura}^{+}$transformants and all of these transformants grew very poorly both on minimal glucose and minimal galactose. In contrast, C. albicans was readily transformed with plasmid pN121I [which encodes the GAL1-regulated mutant $y p t 1$ (N121I) allele]. These pN121I transformants grew normally on minimal glucose, but 10 of 11 transformants tested grew poorly on minimal galactose (Fig. 5). Moreover, plasmid curing experiments verified that growth inhibition in galactose was plasmid-mediated. When four C. albicans $\mathrm{pN} 121 \mathrm{I}$ transformants were cured by culturing for 40 generations in YPD and by plating on FOA medium, four of four FOA-resistant clones derived from each transformant (16 total) exhibited wild-type growth on minimal glucose and minimal galactose media.

\section{Effects of ypt1(N121I) overexpression on protein secretion}

We next examined the abilities of the pYPT1- and pN121I-transformed C. albicans strains to secrete Sap when they were incubated in glucose or galactose liquid media at high cell densities (starting $\mathrm{OD}_{600}$ of 10 ) that allowed minimal cell growth. The cell-free supernatants of pYPT1- and pN121I-transformed C. albicans incubated in glucose-BSA-YE or galactose-BSA-YE for $6 \mathrm{~h}$ contained large amounts of residual BSA. However, after $24 \mathrm{~h}$, the cell-free supernatants of the galactoseincubated pN121I transformants contained much more residual BSA than did the supernatants of the glucoseincubated pN121I transformants or the glucose- or galactose-incubated pYPT1 transformants (Fig. 6). Western blotting showed that the galactose-incubated pN121I transformants secreted much less immuno-
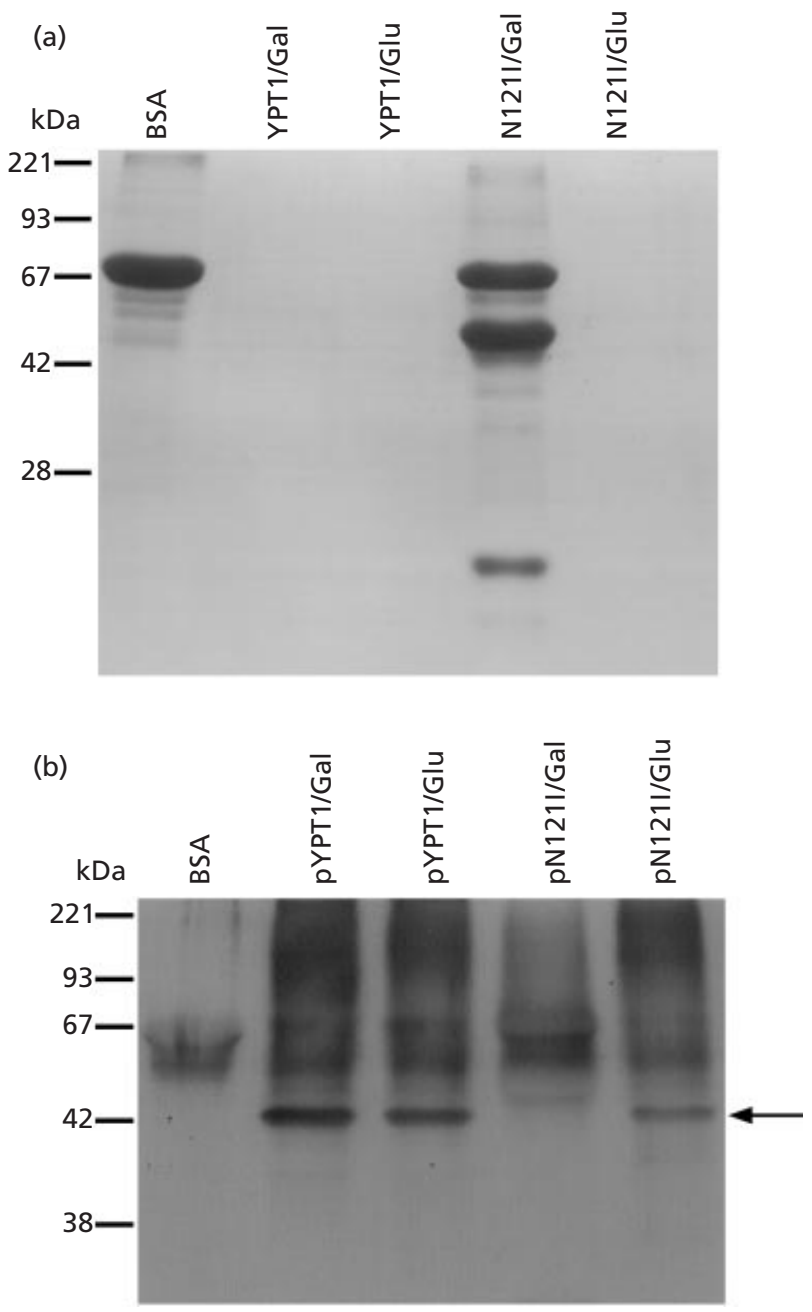

Fig. 6. Effects of ypt1(N121I) overexpression on Sap secretion. (a) Cell-free supernatants were obtained from stationary phase cells and $20 \mu \mathrm{l}$ of each sample was analysed by reducing SDS-PAGE followed by Coomassie blue staining. C. albicans pN121I transformants incubated in galactose-BSA at $30^{\circ} \mathrm{C}$ for $14 \mathrm{~h}$ degraded much less extracellular BSA than pN121I transformants incubated in glucose-BSA or pYPT1 transformants incubated in glucose- or galactose-BSA. (b) Western blot showing that the galactose-incubated pN121I transformants secreted much less immunoreactive Sap (arrow) than glucose-incubated pN121I transformants or pYPT1 transformants incubated in glucose or galactose $(15 \mu \mathrm{l}$ supernatant per lane). The final cell densities at $14 \mathrm{~h}$, as measured by $\mathrm{OD}_{600}$, were $49.4,40.7,13.4$ and 43.3 for pYPT1 transformants in galactose and glucose, and pN121Itransformants in galactose and glucose, respectively.

reactive Sap than glucose-incubated pN121I transformants or pYPT1 transformants in either glucose or galactose.

\section{Effects of ypt1(N121) overexpression on ultrastructural morphology}

There was marked intracellular accumulation of secretory vesicles when a galactose-inhibited pN121I transformant was incubated in minimal galactose for 
(a)
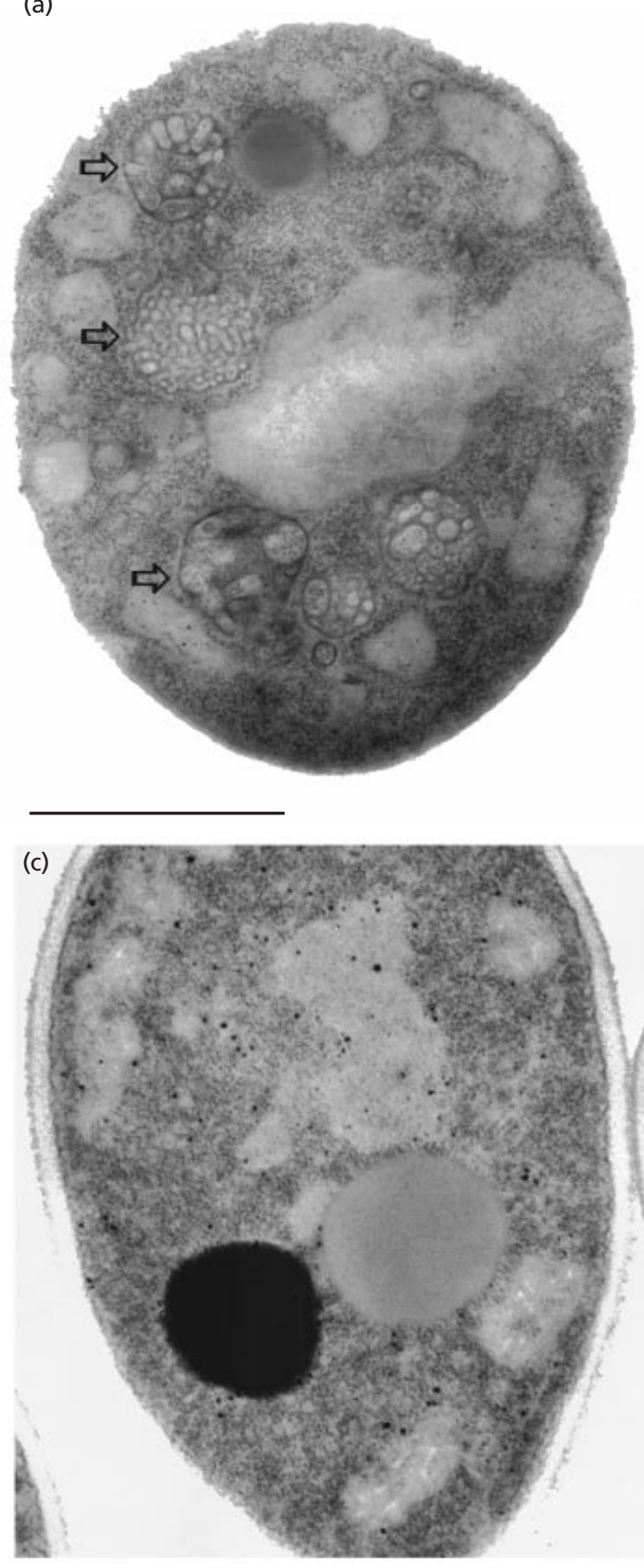
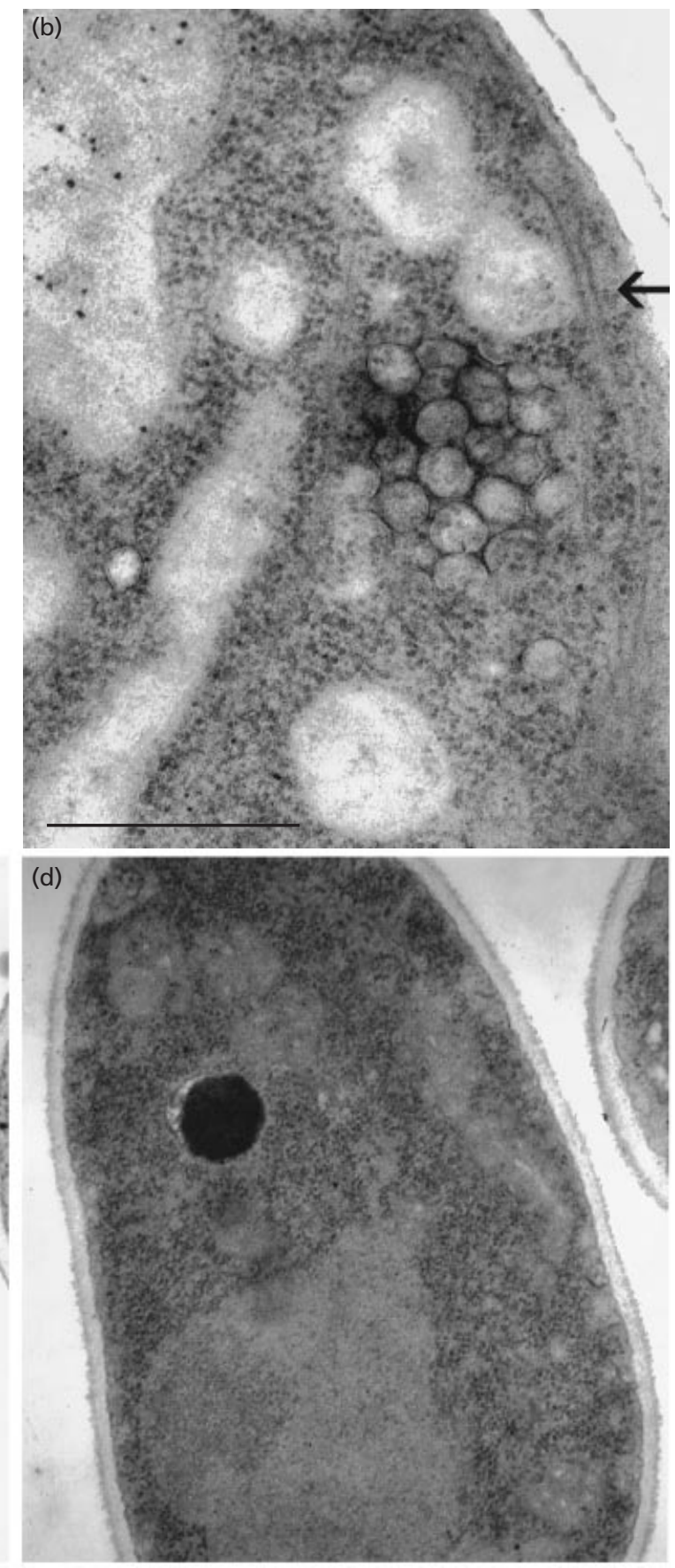

Fig. 7. Effects of ypt1(N121I) overexpression on ultrastructural morphology. Membrane-bound aggregates of vesicles (a, open arrows) and ribbon-like membranous structures (b, arrow) accumulated in pN121I-transformed C. albicans cells incubated in minimal galactose for $6.0 \mathrm{~h}(\mathrm{a}, \mathrm{b})$, but not in pN121I transformants incubated in glucose (c). pYPT1 transformants in galactose (d) or glucose and wild-type C. albicans SC5314 incubated in galactose or glucose did not show abnormal vesicle accumulation (data not shown). Bars, $1 \mu \mathrm{m}(\mathrm{a}) ; 0.5 \mu \mathrm{m}$ (b).

6 h. Many of the vesicles appeared to be closely aggregated within membrane-bound bundle-like structures and a few were elongated with an appearance like ER. In contrast, there was no intracellular accumulation of vesicles in glucose-incubated pN121I transformants, nor in glucose- or galactose-incubated pYPT1 transformants (Fig. 7).

\section{DISCUSSION}

All eukaryotic cells possess a complex apparatus for protein processing and secretion whereby newly synthesized proteins are sequentially transported through a highly organized system of membrane-bound intracellular compartments. After they are synthesized 
in the cytosol, most secreted proteins are transported to the endoplasmic reticulum, through the multiple stacks of the Golgi complex and finally through the plasma membrane. Each step in this pathway requires the donor membrane to form a membrane-bound vesicle by budding followed by fusion to the target membrane, guided by complex interactions between soluble proteins and integral membrane proteins on both donors and targets. This process has been studied extensively in $S$. cerevisiae and more than $30 \mathrm{~S}$. cerevisiae secretion pathway genes have been identified and characterized (Novick et al., 1980; Ferro-Novick \& Novick, 1993). In contrast, only three C. albicans secretion pathway genes (SEC4, SEC14 and SEC18) have been studied (Nieto et al., 1993; Monteoliva et al., 1996; Clement et al., 1998; Mao et al., 1999).

In S. cerevisiae, YPT1 encodes a small ras-like GTPase (Ypt1p) that is required for fusion of pre-Golgi secretory vesicles to the Golgi apparatus (Segev et al., 1988). This protein is found associated with the Golgi and ERGolgi carrier vesicles. Because YPT1 is essential in $S$. cerevisiae, ypt1 null mutants are non-viable. However, temperature-sensitive and dominant-negative mutations in S. cerevisiae YPT1 inhibit growth and protein secretion and cause pre-Golgi secretory vesicles to accumulate intracellularly (Schmitt et al., 1986; Jones et al., 1995; Segev \& Botstein, 1987; Becker et al., 1991; Jedd et al., 1995). In the present study, we cloned the C. albicans YPT1 homologue by screening a $\lambda$ library of C. albicans genomic DNA with a radiolabelled probe based on a C. albicans gene fragment highly homologous to $S$. cerevisiae YPT1 that was identified by the C. albicans genome sequencing project and we showed that C. albicans YPT1 complemented a temperature-sensitive $S$. cerevisiae $y p t 1$ mutant.

Because C. albicans has a diploid genome and no welldefined sexual cycle, classical genetic approaches are of limited use for studying this organism. One powerful experimental approach is to use homologous gene targeting to delete specific C. albicans genes, after which the phenotypic consequences of the resulting mutations can be ascertained (Kelly et al., 1987, 1988; Gorman et al., 1991). A method for homologous gene disruption in a C. albicans ura3s strain (CAI4) whose virulence can be restored to wild-type levels by reintroducing URA3 is now widely used (Fonzi \& Irwin, 1991) and this and related methods have been used to study the effects of several putative C. albicans virulence factors (Ghannoum et al., 1995; Hube et al., 1997; Leidich et al., 1998). However, an important limitation of this general experimental approach is that disruption of essential genes by definition results in non-viability, thereby preventing subsequent investigation of the function of these genes. In the present study, one chromosomal allele of C. albicans YPT1 was readily disrupted by homologous gene targeting. However, efforts to disrupt the second chromosomal YPT1 allele in two different YPT1/ypt1s::hisG strains yielded no viable $y p t 1$ null mutants among a total of 30 transformants analysed. This result suggested that C. albicans YPT1 may be essential, but a firm conclusion on this point based only on inability to generate a null mutant would have required analysis of many more transformants and/or use of a second gene targeting construct with no sequence homology to the first.

Alternative approaches used to study the functions of essential genes include generation of conditional lethal mutants, overexpression of dominant-negative alleles and replacement of a native promoter with a tightly repressible heterologous promoter. Although these approaches are commonly used in S. cerevisiae, they have only recently been used in C. albicans. One group used homologous gene targeting to introduce a temperature-sensitive mutation in the C. albicans homologue of the essential protein myristoylation gene NMT and subtle temperature-dependent differences in gene function were seen (Weinberg et al., 1995). Another group developed a method for using the tightly regulable MET3 promoter to study essential genes in C. albicans (Care et al., 1999). Also, two groups used overexpression of dominant-negative alleles to define the functions of $C$. albicans genes. Feng et al. (1999) use this approach to show that a non-essential ras gene is required for seruminduced hyphal differentiation. Mao et al. (1999) overexpressed a dominant-negative allele of the secretion pathway gene SEC4 to show that this gene is required in C. albicans for growth, protein secretion and fusion of post-Golgi secretory vesicles to the plasma membrane.

Since we found that C. albicans YPT1 was identical to $S$. cerevisiae YPT1 at two positions where known amino acid substitutions (N121I and D124N) encode transdominant inhibitors of $S$. cerevisiae Ypt1p, we used PCR-based site-directed mutagenesis to construct mutant C. albicans ypt1 alleles encoding the analogous amino acid substitutions. Despite multiple repeated attempts, only four pD124N-bearing C. albicans transformants were obtained and they grew too poorly for meaningful analysis. One possible explanation is that the D124N mutant allele may cause stronger dominantnegative effects than the N121I allele. Since the C. albicans GAL1 promoter is incompletely repressed in glucose (Brown et al., 1996), low-level expression of a strong dominant-negative $y p t 1$ allele might have inhibited growth sufficiently to prevent isolation of transformants. Although this could have been tested by replacing the GAL1 promoter with the more tightly repressible MAL2 or MET3 promoters, this was not necessary because numerous transformants were obtained when C. albicans CAI4 was transformed with plasmids encoding the $y p t 1$ (N121I) mutant allele.

The C. albicans pN121I transformants showed poor growth when grown in galactose medium and plasmid curing experiments verified that this effect was mediated by the $y p t 1(\mathrm{~N} 121 \mathrm{I})$ allele. When $\mathrm{pN} 121 \mathrm{I}$ transformants were incubated at high cell density, degradation of Sap was markedly reduced compared to pN121I transformants grown in glucose or pYPT1 transformants in glucose or galactose. These results imply a profound defect in protein secretion, since multiple Saps are normally secreted by C. albicans. Lastly, electron 
microscopy demonstrated marked accumulation of large membrane-bound structures containing many vesicles and some elongated forms. Since some of these structures resembled the elongated ER that accumulates in S. cerevisiae ypt1 mutants (Schmitt, 1988) and since they were very different from the $90-110 \mathrm{~nm}$ spherical post-Golgi vesicles that accumulate in $S$. cerevisiae and C. albicans sec4 mutants (Salminen \& Novick, 1987; Mao et al., 1999), we concluded that the dominantnegative $y p t 1$ allele blocked protein secretion at an early, pre-Golgi stage. Thus, use of a dominant-negative strategy allowed us to ascertain in considerable detail the functions of C. albicans YPT1.

In summary, we have cloned and sequenced the $C$. albicans homologue of the essential $S$. cerevisiae secretory gene YPT1 and we have shown that this gene and its protein product are essential for growth and that they function early in the protein secretory pathway. Furthermore, secretion of the virulence-associated enzyme Sap was markedly reduced when YPT1 function was inhibited by overexpression of a dominant-negative allele. These results confirm the usefulness of dominantnegative inhibitors for studying the functions of essential genes in C. albicans. Lastly, since many virulenceassociated C. albicans proteins are either secreted into the extracellular environment or localize at the cell surface, the general experimental approach used in this study could be used to analyse the post-translational processing and intracellular trafficking of multiple proteins that are involved in virulence and/or pathogenesis.

\section{ACKNOWLEDGEMENTS}

We thank P. T. and B. B. Magee (University of Minnesota) for chromosomal mapping of C. albicans YPT1; Peter Novick (Yale University) for providing strain NY28, plasmid pNB139 and valuable advice; William Fonzi (Georgetown University) for providing strains CAI4 and SC5314, plasmid p5921 and the C. albicans genomic library in $\lambda$ GEM-12; Naga Segev (University of Chicago) for providing S. cerevisiae strain NSY161; Lillemor Wallmark for electron microscopy and photography; and Ann Bartiss for technical advice. Sequence data for C. albicans was obtained from the Stanford DNA Sequencing and Technology Center website at http://www-sequence. stanford.edu/group/candida. Sequencing of C. albicans was accomplished with the support of the NIDR and the Burroughs Wellcome Fund. This work was supported by grants from the Department of Veterans' Affairs (Career Development Award to S.A.L. and Merit Review Award to B.W.) and the National Institute of Allergy and Infectious Diseases (RO1-AI47442).

\section{REFERENCES}

Ausubel, F., Brent, R., Kingston, R., Moore, D., Seidman, J., Smith, J. \& Struhl, K. (1987). Current Protocols in Molecular Biology. New York: Wiley.

Becker, J., Tan, T. J., Trepte, H. \& Gallwitz, D. (1991). Mutational analysis of the putative effector domain of the GTP-binding Ypt1 protein in yeast suggests specific regulation by a novel GAP activity. EMBO J 10, 785-792.
Brennwald, P. \& Novick, P. (1993). Interactions of three domains distinguishing the Ras-related GTP-binding proteins Ypt1 and Sec4. Nature 362, 560-563.

Brown, D. H., Slobodkin, I. V. \& Kumamoto, C. A. (1996). Stable transformation and regulated expression of an inducible reporter construct in Candida albicans using restriction enzyme-mediated integration. Mol Gen Genet 251, 75-80.

Care, R. S., Trevethick, J., Binley, K. M. \& Sudbery, P. E. (1999). The MET3 promoter: a new tool for Candida albicans molecular genetics. Mol Microbiol 34, 792-798.

Chu, W. S., Magee, B. B. \& Magee, P. T. (1993). Construction of a macrorestriction map of the Candida albicans genome. J Bacteriol 175, 6637-6651.

Clement, M., Fournier, H., de Repentigny, L. \& Belhumeur, P. (1998). Isolation and characterization of the Candida albicans SEC4 gene. Yeast 14, 675-680.

Emori, T. G. \& Gaynes, R. P. (1993). An overview of nosocomial infections, including the role of the microbiology laboratory. Clin Microbiol Rev 6, 428-442.

Feng, O., Summers, E., Buo, B. \& Fink, G. (1999). Ras signalling is required for serum-induced hyphal differentiation in Candida albicans. J Bacteriol 181, 6339-6346.

Ferro-Novick, S. \& Novick, P. (1993). The role of GTP-binding proteins in transport along the exocytic pathway. Annu Rev Cell Biol 9, 575-599.

Fonzi, W. A. \& Irwin, M. Y. (1991). Isogenic strain construction and gene mapping in Candida albicans. Genetics 129, 19-24.

Gale, C. A., Bendel, C. M., McClellan, M., Hauser, M., Becker, J. M., Berman, J. \& Hostetter, M. K. (1998). Linkage of adhesion, filamentous growth, and virulence in Candida albicans to a single gene, INT1. Science 279, 1355-1358.

Ghannoum, M. A. (2000). Potential role of phospholipases in virulence and fungal pathogenesis. Clin Microbiol Rev 13, 122-143.

Ghannoum, M. A., Spellberg, B., Saporito-Irwin, S. M. \& Fonzi, W. A. (1995). Reduced virulence of Candida albicans PHR1 mutants. Infect Immun 63, 4528-4530.

Gorman, J. A., Chan, W. \& Gorman, J. W. (1991). Repeated use of GAL1 for gene disruption in Candida albicans. Genetics 129, 19-24.

Hoegl, L., Ollert, M. \& Korting, H. C. (1996). The role of Candida albicans secreted aspartic proteinase in the development of candidoses. Mol Med 74, 135-142.

Hube, B., Sanglard, D., Odds, F. C., Hess, D., Monod, M., Schafer, W., Brown, A. J. \& Gow, N. A. (1997). Disruption of each of the secreted aspartyl proteinase genes SAP1, SAP2, and SAP3 of Candida albicans attenuates virulence. Infect Immun $\mathbf{6 5}$, 3529-3538.

Jarvis, W. R. (1995). Epidemiology of nosocomial fungal infections, with emphasis on Candida species. Clin Infect Dis 20, 1526-1530.

Jedd, G., Richardson, C., Litt, R. \& Segev, N. (1995). The Ypt1 GTPase is essential for the first two steps of the yeast secretory pathway. J Cell Biol 131, 583-590.

Jones, S., Litt, R. J., Richardson, C. J. \& Segev, N. (1995). Requirement of nucleotide exchange factor for Ypt1 GTPase mediated protein transport. J Cell Biol 130, 1051-1061.

Kelly, R., Miller, S. M., Kurtz, M. B. \& Kirsch, D. R. (1987). Directed mutagenesis in Candida albicans: one-step gene disruption to isolate ura3 mutants. Mol Cell Biol 7, 199-208.

Kelly, R., Miller, S. M. \& Kurtz, M. B. (1988). One-step gene 
disruption by cotransformation to isolate double auxotrophs in Candida albicans. Mol Gen Genet 214, 24-31.

Leidich, S. D., Ibrahim, A. S., Fu, Y. \& 8 other authors (1998). Cloning and disruption of caPLB1, a phospholipase B gene involved in the pathogenicity of Candida albicans. J Biol Chem 273, 26078-26086.

Mao, Y., Kalb, V. F. \& Wong, B. (1999). Overexpression of a dominant-negative allele of SEC4 inhibits growth and protein secretion in Candida albicans. J Bacteriol 181, 7235-7242.

Monteoliva, L., Sanchez, M., Pla, J., Gil, C. \& Nombela, C. (1996). Cloning of Candida albicans SEC14 gene homologue coding for a putative essential function. Yeast 12, 1097-1105.

Nieto, A., Sanz, P., Sentandreu, R. \& Agudo, L. D. C. (1993). Cloning and characterization of the SEC18 gene from Candida albicans. Yeast 9, 875-887.

Novick, P. \& Brennwald, P. (1993). Friends and family: the role of the Rab GTPases in vesicular traffic. Cell 75, 597-601.

Novick, P., Field, C. \& Schekman, R. (1980). Identification of 23 complementation groups required for post-translational events in the yeast secretory pathway. Cell 21, 205-215.

Salminen, A. \& Novick, P. J. (1987). A ras-like protein is required for a post-Golgi event in yeast secretion. Cell 49, 527-538.

Sanglard, D., Hube, B., Monod, M., Odds, F. \& Gow, N. (1997). A triple deletion of the secreted aspartyl proteinase genes SAP4,
SAP5, and SAP6 of Candida albicans causes attenuated virulence. Infect Immun 65, 3539-3546.

Schmitt, H. D., Wagner, P., Pfaff, E. \& Gallwitz, D. (1986). The rasrelated YPT1 gene product in yeast: a GTP-binding protein that might be involved in microtubule organization. Cell 47, 401-412.

Schmitt, H. D., Puzicha, M. \& Gallwitz, D. (1988). Study of a temperature-sensitive mutant of the ras-related YPT1 gene product in yeast suggests a role in the regulation of intracellular calcium. Cell 53, 635-647.

Segev, N. \& Botstein, D. (1987). The ras-like yeast YPT1 gene is itself essential for growth, sporulation, and starvation response. Mol Cell Biol 7, 2367-2377.

Segev, N., Mulholland, J. \& Botstein, D. (1988). The yeast GTPbinding YPT1 protein and a mammalian counterpart are associated with the secretion machinery. Cell 52, 915-924.

Staab, J. F., Bradway, S. D., Fidel, P. L. \& Sundstrom, P. (1999). Adhesive and mammalian transglutaminase substrate properties of Candida albicans Hwp1. Science 283, 1535-1538.

Weinberg, R. A., McWherter, C. A., Freeman, S. K., Wood, D. C., Gordon, J. I. \& Lee, S. C. (1995). Genetic studies reveal that myristoylCoA:protein $\mathrm{N}$-myristoyltransferase is an essential enzyme in Candida albicans. Mol Microbiol 15, 241-250.

Received 5 January 2001; revised 21 February 2001; accepted 5 March 2001. 